\title{
Effect of using microwaves for catalysts preparation on the catalytic acetalization of glycerol with furfural to obtain fuel additives
}

https://doi.org/10.1515/chem-2018-0047

received September 30, 2017; accepted March 14, 2018.

\begin{abstract}
The effect of using microwaves or conventional heating for synthesizing/modifying several acid micro-and mesoporous materials have been studied by testing them as catalysts for the acetalization of glycerol with furfural to obtain 1,3-dioxane and 1,3-dioxolane (fuel additives). Microwaves allowed us the incorporation of higher amounts of sulfonic groups in SBA-15 and montmorillonite MK-10 catalysts whereas for zeolite samples the differences were not significant. The catalysts prepared/modified with microwaves led to higher conversion but slight lower selectivity to the desired products than those prepared by conventional heating. Sulfonated montmorillonite MK-10 catalysts showed high conversion (62-68\%) and the highest selectivity values to the desired products $(72-86 \%)$. This has been related to the higher surface density of Brønsted acid sites observed for these catalysts. The presence of Lewis acid centres in the $\mathrm{ReO}_{x} /$ hectorite catalyst increased the formation of dioxane.
\end{abstract}

Keywords: glycerol; microwaves; furfural; acetalization; fuel additives.

\section{Introduction}

Conversion of biomass into functionalized platform molecules, such as sugars (glucose, xylose), polyols, furans (furfural, 5-hydroxymethylfurfural) and acids allows for

\footnotetext{
*Corresponding author: Yolanda Cesteros, Universitat Rovira i Virgili, Departament de Química Física i Inorgànica C/ Marcel•lí Domingo, 1, 43007 Tarragona, Spain, E-mail: yolanda.cesteros@urv.cat Esther Gutiérrez-Acebo, Federico Guerrero-Ruiz, Mélanie Centenero, Jordan S. Martínez, Pilar Salagre: Universitat Rovira i Virgili, Departament de Química Física i Inorgànica C/ Marcel•lí Domingo, 1, 43007 Tarragona, Spain
}

the production of a wide range of fuels and chemicals [1-2]. The development of technologies to produce energy and chemicals from renewable resources, as alternative to petroleum-derived products, has prompted biomass valorization to become an important area of research as a means to establish a sustainable economy [3]. Lignocellulose, the most abundant renewable biomass, is considered the main raw material on a biorefinery concept, since its non-edible nature does not compete with food crops and is less expensive than conventional agricultural feedstocks. Appropriate upgrading strategies using catalytic technologies are used for conversion of biomass into valuable products. Furfural, a versatile key derivative produced from pentosan-rich biomass, constitutes an important building block for the production of bio-based chemicals.

The catalytic acetalization of furfural with glycerol is becoming an attractive process for the production of a mixture of cis and trans isomers of five- and six-membered furans called dioxolane and dioxane, respectively (Figure 1). Glycerol acetals and ketals are extensively used as additives, bases, scents and flavours in several industries. Particularly, 1,3-dioxane and 1,3-dioxolane ((2-(furan-2-yl)-1,3-dioxane-5-ol) and (2-(furan-2-yl)1,3-dioxolane-4-yl)methanol), respectively) have been described as additives of biodiesel fuels since they can improve the viscosity and cold properties of biodiesel, and have reasonable oxidation stability and flash point. Moreover, significant reduction of hydrocarbons, carbon monoxide and particulate matter emissions can be achieved by adding these oxygenated compounds to the standard diesel fuel [4]. Furthermore, this reaction is a challenging option for the revalorization of glycerol, obtained as surplus during biodiesel production.

Traditionally, homogeneous Brønsted acids, such as $\mathrm{HCl}, \mathrm{H}_{2} \mathrm{SO}_{4}, \mathrm{H}_{3} \mathrm{PO}_{4}$ and p-toluenesulfonic acid, have been employed for this reaction [5]. However, such procedures present several limitations associated to product 


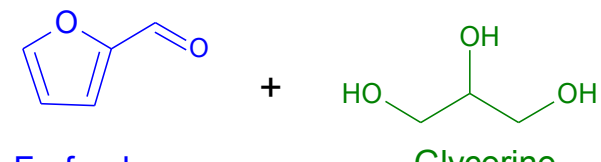

Furfural
Glycerine
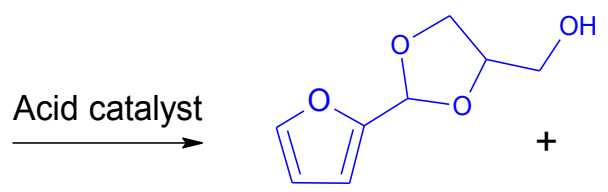

1,3-dioxolane

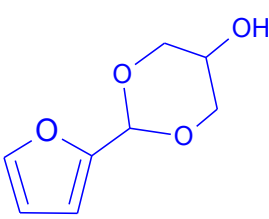

1,3-dioxane

Figure 1: Scheme of the acetalization of glycerol with furfural.

separation, catalyst recovery, equipment corrosion, and generation of large amounts of wastes. To overcome these drawbacks, focus has recently shifted on the development of renewable and environmentally benign heterogeneous catalytic systems.

A variety of heterogeneous catalysts, such as ionexchange resins, zeolites, heteropolyacids, and promoted metal oxides, have been tested for the acetalization of glycerol with different carbonyl compounds. However, there are few references for the acetalization of glycerol with furfural [2,6-8]. Mallesham et al. checked several Moand $\mathrm{W}$-promoted solid acids under solvent free conditions at room temperature [6]. These authors found that the addition of $\mathrm{Mo}^{6+}$ ions to $\mathrm{SnO}_{2}$ increased its activity due to the presence of higher amounts of acid sites, higher BET area, lattice defects and redox properties. Thus, 51, 67 and 75\% of glycerol conversion values were obtained when using $\mathrm{SnO}_{2}, \mathrm{WO}_{3} / \mathrm{SnO}_{2}$ and $\mathrm{MoO}_{3} / \mathrm{SnO}_{2}$ catalysts, respectively. FTIR studies of adsorbed pyridine showed the existence of a larger number of Brønsted acid sites compared to Lewis acid sites in these catalysts. Furthermore, the use of nitrogen flow during reaction circumvented water inhibition and enabled high yields of acetal (80-90\%) under nearly stoichiometric furfural to glycerol molar ratio using homogeneous $\mathrm{ZnCl}_{2}$ and heterogeneous Al-MCM-41 catalyst [7]. More recently, Konwar et al. reported the use of lignosulfonate-based macro/mesoporous solid catalysts for the acetalization of glycerol with acetone, furfural and methyl levulinate [8]. These sulfonated carbons, with high amounts of strong Brønsted acid sites, resulted in high conversion of glycerol ( $\geq 93 \%$ ) without observing the formation of byproducts [8]. There are still many points to study in this reaction like understand the effect of the different type, strength and amount of acid sites on the catalytic activity.

The use of microwaves for the synthesis or modification of catalysts considerably decreased the preparation times, with the subsequent energy saving [9-11], following one of the principles of Green Chemistry. Microwave also modifies the samples properties, which can be of interest in catalysis.
The aim of this work was to study the effect of using microwaves versus conventional heating for the preparation of several acid-modified micro- and mesoporous materials catalysts on the acetalization of glycerol with furfural to obtain fuel additives.

\section{Experimental}

\subsection{Preparation of catalysts}

Two commercial zeolites and one commercial montmorillonite K-10 were sulfonic acid-functionalized using microwaves (Milestone ETHOS-TOUCH CONTROL equipped with a temperature controller) and conventional heating for comparison. Specifically, $2 \mathrm{~g}$ of zeolite Na-Beta (B) (Zeochem, $\mathrm{Si} / \mathrm{Al}=10), 2 \mathrm{~g}$ of Na-mordenite (M) (Zeolyst, $\mathrm{Si} / \mathrm{Al}=6.5$ ), and $2 \mathrm{~g}$ of montmorillonite K-10 (MK-10) (SigmaAldrich, $\mathrm{Si} / \mathrm{Al}=2.7$ ) were treated, respectively, with $1.4 \mathrm{~g}$ of chlorosulfonylphenylethyltrimethoxysilane (CSPTMS, 50 wt \% in methylene chloride, Gelest) in $150 \mathrm{~mL}$ of $2 \mathrm{M}$ $\mathrm{HCl}$ by refluxing with microwaves at $40^{\circ} \mathrm{C}$ for $2 \mathrm{~h}$ (BSMw, MSMw, MK-10-SMw) or by conventional heating at $40^{\circ} \mathrm{C}$ for $2 \mathrm{~h}$ (BSC, MSC, MK-10-SC).

Furthermore, two sulfonated SBA-15 catalysts, with a molar composition of $1.2 \mathrm{SiO}_{2} / 0.2 \mathrm{CSPTMS} / 6.5 \mathrm{HCl} / 180 \mathrm{H}_{2} \mathrm{O}$ were prepared following the method reported elsewhere [12]. The sulfonation step was made by refluxing at $40^{\circ} \mathrm{C}$ for $2 \mathrm{~h}$ using microwaves (sample SBA-15-SMw) or by refluxing at $40^{\circ} \mathrm{C}$ for $24 \mathrm{~h}$ (sample SBA-15-SC). The corresponding reaction mixtures were heated in an autoclave at $100^{\circ} \mathrm{C}$ for $24 \mathrm{~h}$. The surfactant template was removed by extraction with ethanol, and later calcination at $200^{\circ} \mathrm{C}$ for $24 \mathrm{~h}$.

Hectorites are smectites with weak Brønsted acidity. Two hectorites were prepared following the procedure described elsewhere [13] consisting of an initial slurry (3 wt $\%$ solids) containing $\mathrm{SiO}_{2}$, fresh brucite $\mathrm{Mg}(\mathrm{OH})_{2}$, and $\mathrm{LiF}$, following the molar ratio $7.5 \mathrm{SiO}_{2}: 3 \mathrm{Mg}(\mathrm{OH})_{2}: 1 \mathrm{LiF}$, which was stirred vigorously for $1 \mathrm{~h}$. Fresh brucite was previously synthesized by precipitation of a $10 \mathrm{M}$ ammonia solution 
onto $\mathrm{MgCl}_{2}$ (aq). Then, one sample was aged in autoclave with microwaves at $120^{\circ} \mathrm{C}$ for $8 \mathrm{~h}$ (HectMw), and the other one was aged in autoclave by conventional heating at $120^{\circ} \mathrm{C}$ for 6 days (HectC). $0.5 \mathrm{~g}$ of both samples were exchanged by refluxing with $50 \mathrm{~mL}$ of $1 \mathrm{M} \mathrm{NH}_{4} \mathrm{NO}_{3}$ aqueous solution for at $100^{\circ} \mathrm{C}$ for $1 \mathrm{~h}$. Then, samples were filtered, washed with deionized water, dried overnight and calcined at $350^{\circ} \mathrm{C}$ for $6 \mathrm{~h}$ to obtain the protonated forms (H-HectC and $\mathrm{H}-\mathrm{HectMw})$.

In order to observe the effect of Lewis acidity, one ReHectMw was prepared by impregnating $0.5 \mathrm{~g}$ of sample HectMw with a $0.5 \mathrm{M}\left(\mathrm{NH}_{4}\right) \mathrm{ReO}_{4}$ solution. The dried sample was calcined at $300^{\circ} \mathrm{C}$ for $5 \mathrm{~h}$.

\subsection{Characterization of catalysts}

Powder X-ray diffraction patterns of the samples were obtained with a Siemens D5000 diffractometer using nickel-filtered $\mathrm{CuK} \alpha$ radiation. Samples were dusted on double-sided sticky tape and mounted on glass microscope slides. The patterns of zeolite, montmorillonite and hectorite samples were recorded over a range of $2 \Theta$ angles from $5^{\circ}$ to $40^{\circ}$ while the patterns of SBA- 15 samples were recorded between $2 \Theta$ angles from 0.5 o to 10 ㅇ. Crystalline phases were identified using the Joint Committee on Powder Diffraction Standards (JCPDS) files (43-0171, 480074, 03-0016, 03-0168) corresponds to mordenite, beta, montmorillonite and hectorite, respectively. Crystallinity of the modified mordenites was determined by comparing the sum of the peak areas of (150), (202), (350) and (402) (22-32ㅇ 20) with respect to commercial Na-mordenite. The integrated intensity of the signal at $2 \theta=22.4^{\circ}$ was used to evaluate the crystallinity of beta samples.

BET areas were calculated from nitrogen adsorption isotherms obtained at $77 \mathrm{~K}$ using a Quadrasorb SI surface analyser with a value of $0.164 \mathrm{~nm}^{2}$ for the cross-section of the nitrogen molecule. Zeolite samples were pretreated in vacuum at $300^{\circ} \mathrm{C}$ for $6 \mathrm{~h}, \mathrm{SBA}-15$ at $150^{\circ} \mathrm{C}$ for $12 \mathrm{~h}$ and the rest of samples at $80^{\circ} \mathrm{C}$ for $12 \mathrm{~h}$.

Thermogravimetric analyses (TGA) of sulfonated catalysts were performed with a TA instruments equipment from $50^{\circ} \mathrm{C}$ to $800^{\circ} \mathrm{C}$ at $10^{\circ} \mathrm{C} / \mathrm{min}$ under airflow in order to determine the amount of sulfonic groups incorporated in the samples. Additionally, the acid capacity of sulfonated catalysts was measured through the determination of cation-exchange capacities using aqueous sodium chloride (2M) solutions as cationic-exchange agent. Released protons were then potentiometrically titrated [14].
Table 1: Characterization of the sulfonated catalysts.

\begin{tabular}{lllll}
\hline Catalysts & $\begin{array}{l}\text { Cristallinity } \\
(\%)(X R D)^{a}\end{array}$ & $\begin{array}{l}\text { BET area } \\
\left(\mathrm{m}^{2} / \mathrm{g}\right)\end{array}$ & $\begin{array}{l}\text { Sulfur } \\
\text { content }^{\mathrm{b}}\end{array}$ & $\begin{array}{l}\text { Acid capacity } \\
(\mathrm{meq} \mathrm{H}\end{array}$ \\
\hline B & 100 & 603 & --- & --- \\
BSC & 29 & 330 & 0.74 & 0.75 \\
BSMw & 43 & 486 & 0.70 & 0.72 \\
M & 100 & 353 & --- & $\cdots$ \\
MSC & 21 & 391 & 0.68 & 0.72 \\
MSMw & 32 & 362 & 0.72 & 0.77 \\
MK-10 & --- & 202 & --- & --- \\
MK-10-SC & --- & 86 & 0.51 & 0.54 \\
MK-10-SMw & --- & 82 & 0.71 & 0.77 \\
SBA-15-SC & --- & 654 & 0.83 & 0.35 \\
SBA-15-SMw & --- & 572 & 1.01 & 0.75 \\
\hline
\end{tabular}

a Calculated from XRD patterns;

${ }^{b}$ (mmol organic sulfonic acid group/g sample) calculated from TGA; c Obtained by potentiometric titration.

\subsection{Catalytic Activity}

The catalytic reaction was carried out for all catalysts in a batch reactor $(50 \mathrm{~mL}$ amber three necked round bottom flask) with glycerol: furfural ratio 1:1, 40으, $2 \mathrm{~h}$, and $5 \mathrm{wt}$ $\%$ of catalyst. Nitrogen flow was bubbled during reaction in order to decrease the byproducts formation. Reaction products were analyzed using a gas chromatograph (Shimadzu GC2010A) with FID detector equipped with a TRB-WAX column. Calibration lines were applied to calculate glycerol conversion and selectivity to the reaction products using butanol as internal standard.

Ethical approval: The conducted research is not related to either human or animals use.

\section{Results and Discussion}

\subsection{Characterization of the catalysts}

XRD patterns of the sulfonated zeolite samples showed that, after modification, the materials maintained their structure (Figure 2a and b) although some decrease of crystallinity was detected for all of them, in less extent for the corresponding microwaved samples (Table 1). Sulfonated montmorillonite MK-10 samples also maintained the structure of the commercial starting 

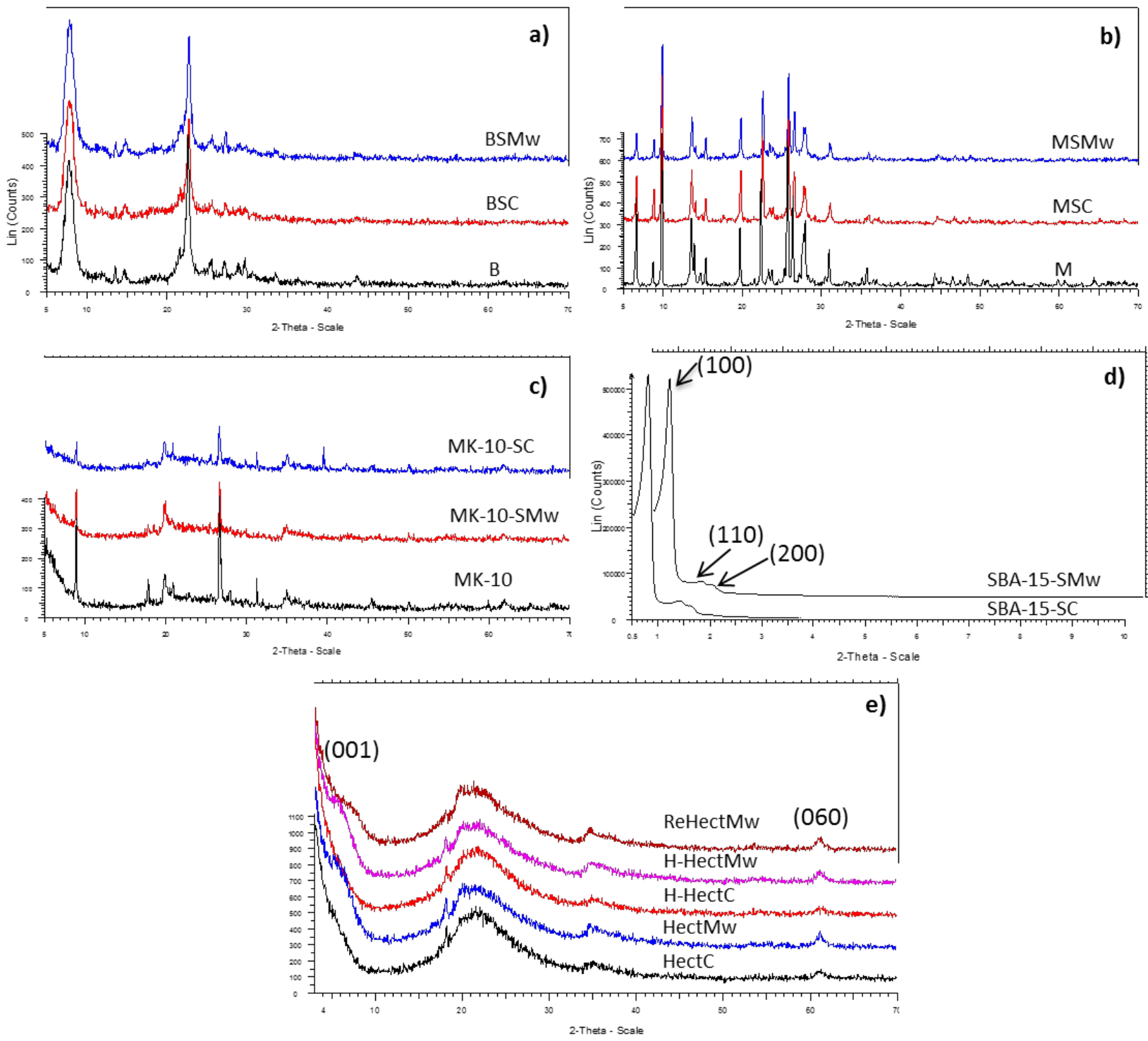

Figure 2: XRD patterns of the catalysts.

material (Figure 2c) while XRD patterns of SBA-15 samples showed their typical hexagonal structure with the characteristic peaks corresponding to (100), (110) and (200) reflections (Figure 2d). Finally, XRD of the hectorite samples were typical of clay materials showing the phase corresponding to hectorite (Figure 2e). However, 001 reflection, related to layer stacking, was not well defined. This indicates a low degree of order stacking although this reflection appeared a bite more defined for the samples prepared with microwaves. This confirms that the use of microwaves considerably reduced the crystallization time. In contrast, 060 reflection, related to the crystallinity of the layers appeared enough well defined for all samples. Additionally, a broad band around 22.8o corresponding to amorphous silica was also observed in all cases. Modification by protonation or impregnation with Re did not practically change the crystallinity of the samples. Nitrogen adsorption-desorption isotherms were type I for all the zeolite and hectorite samples, attributed to microporous materials, and type IV for montmorillonite MK-10 and SBA-15 samples, corresponding to mesoporous materials. Montmorillonite is a microporous layered clay of the smectite group with general formula $\left[\mathrm{Si}_{8}\left(\mathrm{Al}_{4 \times \mathrm{x}} \mathrm{Mg}_{\mathrm{x}}\right)\right.$ $\left.(\mathrm{OH})_{4} \mathrm{O}_{20}\right] \mathrm{M}_{\mathrm{n}+\mathrm{x} / \mathrm{n}} \cdot \mathrm{mH}_{2} \mathrm{O}$. However, montmorillonite MK-10 is commercially obtained from natural montmorillonite by acid treatment resulting in the appearance of mesoporosity. 
A decrease of specific surface area was observed after sulfonation for beta catalysts (Table 1) while sulfonated mordenite catalysts showed higher surface area than their parent mordenite. Zeolite beta has a three-dimensional 12-ring pore system (straight channels of diameter $6.6 \mathrm{x}$ $6.7 \AA$ and sinusoidal channels of diameter 5.6 x $5.6 \AA$ ) and, because of this property, the framework is very flexible. In contrast, mordenite has one-dimensional pore system with main channels of diameter 6.7 x 7.0 A and compressed channels of diameter $2.6 \times 5.7 \AA$. The decrease of surface area observed for sulfonated beta samples could be mainly attributed to the easier introduction of the sulfonic groups in this more flexible zeolite framework. On the other hand, the higher specific surface areas observed for the sulfonated mordenites with respect to the commercial mordenite could be associated to the loss of aluminium in the zeolite structure, due to the acidic medium used during sulfonation, which results in higher mesoporosity, as reported before for partially dealuminated mordenites [9].

For the sulfonated montmorillonite K-10 samples, the decrease of surface area can be explained because of the introduction of the sulfonic groups, which can partially occupy the pores. SBA-15 samples showed the highest surface area values, as expected, due to their higher mesoporosity (Table 1).

For hectorite samples, the use of microwaves resulted in slight lower surface area (Table 2). This could be related to the slight higher crystallinity observed for this sample by XRD. The presence of Re did not practically affect the surface area of the hectorite sample (Table 2).

TGA results of the sulfonated samples allowed us to conclude that microwaves led to the incorporation of higher amounts of sulfonic groups in SBA-15 and montmorillonite MK-10 catalysts whereas for zeolite samples the differences were not significant. These results were confirmed regarding the acid capacity values of the zeolite samples (Table 1).

\subsection{Catalytic Activity results}

All catalysts were active for the acetalization of glycerol with furfural (Figures 3 and 4). The main reaction product was dioxolane, in all cases. The other product of interest, dioxane, was also obtained but in lower amounts with the exception of catalyst ReHectMw, as explained below. Condensation products were also detected by gas chromatography for all catalysts at higher retention times than the desired products.
Table 2: BET surface area of the hectorite catalysts.

\begin{tabular}{llllll}
\hline Catalyst & HectC & H-HectC & HectMw & H-HectMw & ReHectMw \\
\hline $\begin{array}{l}\text { BET area } \\
\left(\mathrm{m}^{2} / \mathrm{g}\right)\end{array}$ & 154 & 151 & 148 & 146 & 143 \\
\hline
\end{tabular}

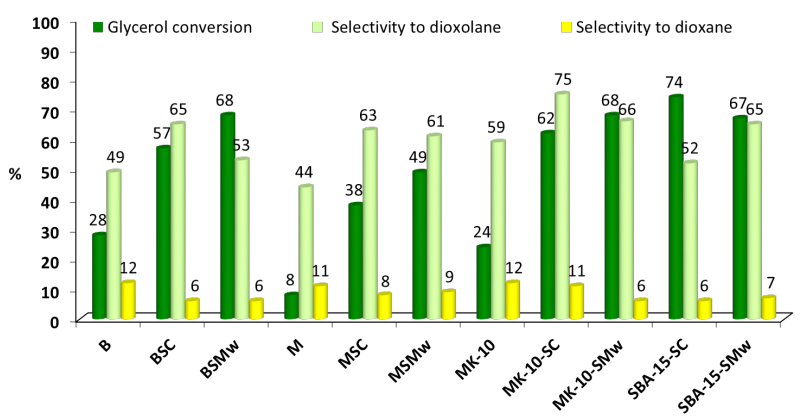

Figure 3: Catalytic activity results of the sulfonated catalysts.

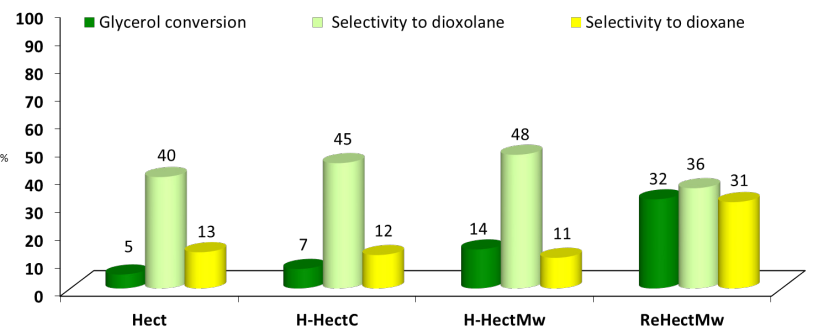

Figure 4: Catalytic activity results of the hectorite catalysts.

The incorporation of the sulfonic groups in beta, mordenite, and montmorillonite-K-10 catalysts resulted in higher conversion, higher selectivity to dioxolane, due to the presence of higher amounts of stronger Brønsted acid sites, and lower selectivity to dioxane. The higher selectivity to dioxane observed for the commercial materials could be probably attributed to the presence of higher amounts of Lewis acid sites in these samples $\left(\mathrm{Na}^{+}\right)$. This was later confirmed taking into account the catalytic results of all the catalysts prepared.

Sulfonated zeolites led to moderate values of conversion (38-68\%) and moderate selectivity to the products of interest (59-71\%). The lower conversion values for mordenite catalysts with respect to beta catalysts can be related to the different pore size distribution and the lower external surface area of mordenite catalysts the framework of which has a one-dimensional pore system, as previously commented.

Sulfonated SBA-15 catalysts resulted in higher conversion $(67-74 \%)$, but similar selectivity to dioxane + dioxolane $(58-72 \%)$ than modified zeolites. Sulfonated montmorillonites MK-10 showed high conversion and 
the highest selectivity values to the desired products $(72-86 \%)$. This can be related to the higher surface density of Brønsted acid sites present in these catalysts (meq $\mathrm{H}^{+} /$ $\mathrm{m}^{2}$ ) (Table 1).

H-Hectorites showed the lowest conversion values (Figure 3). This can be related to the weak acidity of these materials, even protonated, as widely reported in the literature. Interestingly, the catalyst with Re, prepared with the non-protonated form of HectMw in order to decrease the presence of Brønsted acid sites, exhibited the maximum formation of dioxane (31\%). Therefore, the presence of Lewis acid sites favoured the formation of dioxane, and both Brønsted and Lewis acid sites were active for this reaction.

On the whole, the catalysts prepared with microwaves led to higher conversion but slight lower selectivity to the desired products than those prepared by conventional heating except in the case of SBA-15, which showed an opposite effect. The slight catalytic differences observed between the zeolites sulfonated with microwaves with respect to their counterparts synthesized by conventional heating can be related to the similar amount, strength and accessibility of their acid sites (Table 1). For sulfonated montmorillonite MK-10 and sulfonated SBA-15 catalysts, which exhibited higher differences in the amount of acid sites when prepared with microwaves or by conventional heating (Table 1), the slight differences observed in their catalytic activity indicates that not all the acid sites generated by sulfonation were accessible to the reagents for the corresponding microwaved samples.

The catalytic life of the two sulfonated montmorillonite MK-10 catalysts was evaluated in three consecutive runs under the same reaction conditions (Figure 5). After each run, catalyst was recovered by centrifugation, washed in ethanol at room temperature and dried. We observed similar conversion and a progressive loss of selectivity to the desired products, especially to dioxalane, for both catalysts. Interestingly, the decrease of selectivity was slight higher for the catalyst sulfonated by conventional heating. This confirms that the use of microwaves during sulfonation involved some differences in the properties of the final catalysts.

\section{Conclusions}

Microwaves allowed the incorporation of higher amounts of sulfonic groups in SBA-15 and montmorillonite MK-10 catalysts. Both Brønsted and Lewis acid sites were active for this reaction. The catalysts prepared/modified with

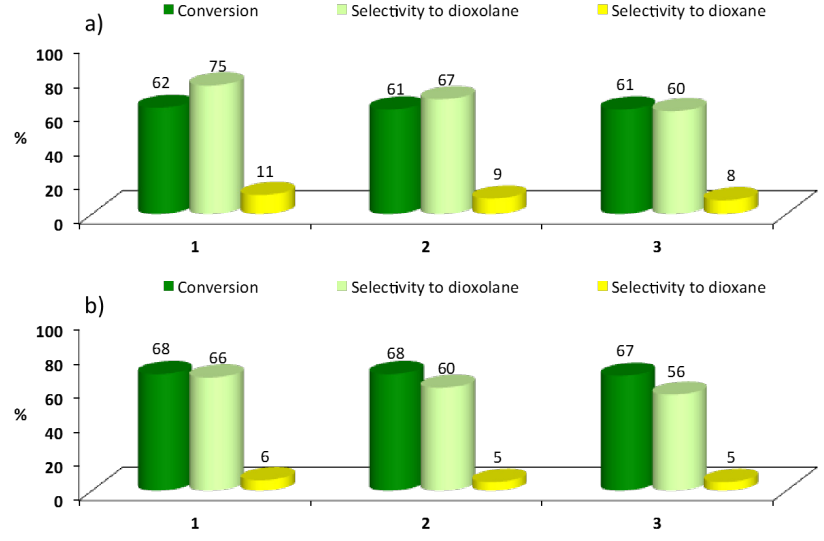

Figure 5: Reuse studies of catalysts a) MK-10-SC and b) MK-10-SMw.

microwaves led to higher conversion but slight lower selectivity to the desired products than those prepared/ modified by conventional heating. The higher surface density of Brønsted acid sites could explain the best catalytic behavior observed in montmorillonite MK-10 catalysts (62-68\% of conversion, $72-86 \%$ selectivity to the desired products). Lewis acidity favoured the formation of dioxane, as deduced from the incorporation of $\mathrm{ReOx}$ species in a hectorite catalyst with weak Brønsted acidity.

Acknowledgments: Authors acknowledge the financial support of the Ministerio de Economía y Competitividad of Spain and Feder Funds (CTQ2015-70982-C3-3-R), and the recognition from the Generalitat de Catalunya (2014 SGR 1146).

Conflict of interest: Authors state no conflict of interest.

\section{References}

[1] Sheldon R.A., Green and sustainable manufacture of chemicals from biomass: state of the art, Green Chem., 2014, 16, 950963.

[2] Climent M.J., Corma A., Iborra S., Conversion of biomass platform molecules into fuel additives and liquid hydrocarbon fuels, Green Chem., 2014, 16, 516- 547.

[3] Alonso D.M., Bond J.Q., Dumesic J.A., Catalytic conversion of biomass to biofuels, Green Chem., 2010, 12, 1493-1513.

[4] Nair G.S., Adrijanto E., Alsalme A., Kozhevnikov I.V., Cooke D.J., Brown D.R., Shiju N.R., Glycerol utilization: solvent-free acetalisation over niobia catalysts, Catal. Sci. Technol., 2012, 2, 1173-1179.

[5] Umbarkar B., Kotbagi T.V., Biradar A.V., Pasricha R., Chanale J., Dongare M.K., Mamede A.S., Lancelot C., Payen E., Acetalization of glycerol using mesoporous $\mathrm{MoO}_{3} / \mathrm{SiO}_{2}$ solid acid catalyst, J. Mol. Catal. A: Chem., 2009, 310, 150-158. 
[6] Mallesham B., Sudarsanam P., Raju G., Reddy B.M., Design of highly efficient Mo and W-promoted $\mathrm{SnO}_{2}$ solid acids for heterogeneous catalysis: acetalization of bio-glycerol, Green Chem., 2013, 15, 478-489.

[7] Wegenhart B.L., Liu S., Thom M., Stanley D., Abu-Omar M.M., ACS Catal., 2012, 2, 2524-2530.

[8] Konwar L.J., Samikannu A., Mäki-Arvela P., Boström D., Appl. Catal. B: Environ., 2018, 220, 314-323.

[9] González M.D., Cesteros Y., Salagre P., Comparison of dealumination of zeolites beta, mordenite and ZSM- 5 by treatment with acid under microwave irradiation, Micropor. Mesopor. Mater., 2011, 144, 162-170.

[10] Sánchez T., Salagre P., Cesteros Y., Ultrasounds and microwaveassisted synthesis of mesoporous hectorites, Micropor. Mesopor. Mater., 2013, 171, 24-34.

[11] Gebretsadik F.B., Salagre P., Cesteros Y., Use of polymer as template in microwave synthesis of saponite. Study of several factors of influence, Appl. Clay Sci., 2013, 87, 170-178.

[12] Zhao W., Salame P., Launay F., Gédéon A., Hao Z., J. Porous Mater., 2008, 15, 139.

[13] Vicente I., Salagre P., Cesteros Y., Phys. Proced., 2010, 8, 88 93.

[14] Melero J.A., Stucky G.D., van Grieken R., Morales G., J. Mater. Chem., 2002, 12, 1664. 